\title{
Communication using Ultra Wide-band Pulse Position Modulation for In-body Sensors
}

\author{
Anna N. Kim ${ }^{*} \quad$ Pål A. Floor ${ }^{1,2}$ Tor A. Ramstad ${ }^{2}$ llangko Balasingham ${ }^{1,2}$ \\ ${ }^{1}$ Intervention Center \\ Oslo University Hospital and Institute of Clinical Medicine NO-0027, Oslo, Norway \\ ${ }^{2}$ Dept. of Electronics and Telecommunications \\ Norwegian University of Science and Technology NO-7491, Trondheim, Norway \\ annak@ieee.org andflo@rr-research.no ramstad@iet.ntnu.no ilangkob@medisin.uio.no
}

\begin{abstract}
One important challenge in medical in-body sensors today is robust transmission to on-body receivers via wireless channels. The miniature in-body sensors are often restricted in physical size and have limited power supply. At the same time, wireless transmission must also cope with large attenuation due to body tissues and organs. Applications such as the capsule endoscope, require high data rates in order to transmit colour video from inside the small intestine. For such applications, ultra wideband (UWB) communication with pulse-position modulation (PPM) is a suitable solution. In this paper we present a complete communication chain for a capsule endoscope with PPM for transmission over multipath channels. Through simulation we evaluate the impact of channel coding and interleaving, channel state estimation and combining, and estimation of the required transmit power, using a realistic channel model.
\end{abstract}

\section{Categories and Subject Descriptors}

H.4.3 [Information Systems Applications]: Communications Applications

\section{Keywords}

ultra wideband, pulse-position modulation, in-body communication, multipath channels, transmission power estimation

\section{INTRODUCTION}

Ultra wideband (UWB) is a form of radio communication that utilizes a large bandwidth, typically larger than 500 MHz. Compared to typical narrow band communication, UWB has some unique features such as very low transmit power, high data rate, and better resistance to multipath fading. Because it is able to operate below the noise floor,

${ }^{*}$ This work was supported by the Research Council of Norway (NFR), under the project MELODY nr. 187857/S10

\footnotetext{
Permission to make digital or hard copies of all or part of this work for personal or classroom use is granted without fee provided that copies are not made or distributed for profit or commercial advantage and that copies bear this notice and the full citation on the first page. To copy otherwise, to republish, to post on servers or to redistribute to lists, requires prior specific permission and/or a fee.

Copyright 20XX ACM X-XXXXX-XX-X/XX/XX ...\$10.00.
}

UWB communication can co-exist with narrow band users without causing much interference. Its short communication range (up to $10 \mathrm{~m}$ in air) also makes efficient indoor spectrum reuse a possibility. These characteristics make UWB technology particularly attractive for health care and medicine and research in this area has gained high momentum since FCC unlicensed the use of UWB in 2002.

For in-body devices, low power consumption is an especially important design factor. The capsule endoscope is one such example. To examine inner body cavities like the small intestine, the patient swallows a pill sized capsule which contains a miniature camera, a light source, battery, processing and transmitting units. The capsule relies on the natural movement of the inner organs to pass through the body while taking photos along the way. These images are transmitted wirelessly to on-body receivers, and then further processed for diagnosis. Because of the restriction in size, the capsule endoscope has very limited power supply. Since it is designed to operate over 8 to 10 hours, all processes carried out within the endoscope must be extremely power efficient. At the same time, high quality video also requires a high data transmission rate. Taking all these obstacles into account, UWB becomes a suitable choice of communication for this scenario.

One way to effectively exploit the large bandwidth provided by UWB is to apply pulse position modulation (PPM) [1]. To transmit $M$ bits using PPM, a very short duration pulse is transmitted in one of $2^{M}$ possible time-shifts (positions) within the symbol duration. Compared to binary transmission, for example on-off keying (OOK), $2^{M}$-PPM uses $2^{M} / M$ times more bandwidth for transmitting the same amount of data. The benefit, comes from the greatly reduced transmit power, since only a single pulse is used for transmitting $M$ bits. The larger $M$ is, the lower the average transmit power can be pushed. At the same time, higher level PPM also means better BER performance. In fact, if we place no restriction on bandwidth, it can be shown that PPM is optimal in the information theoretical sense as the bandwidth and $2^{M} \rightarrow \infty[1$, Chapter 8$]$. This motivates the use of PPM in UWB applications.

In-body to on-body communication using UWB is however, a challenging problem. In order to design and implement an effective communication strategy, the wireless channel must be accurately modeled. The human tissue absorbs and attenuates the wireless signal to a much greater degree than free space. Depending on the position of the in-body capsule, channel characteristics also vary. In [3], an UWB 
link operating at 1-6 GHz was characterized at various depth for the abdominal region. An alternative channel model for UWB communication for the capsule endoscope was proposed in [4]. There, the on-body receivers are placed in the form of a belt. Height and angle of capsule in-body position relative to the receivers were used to parameterize the channel model. More recently, another propagation model for the capsule endoscope application was presented in [2], using the finite-difference time-domain (FDTD) method. Note that characteristics of channel models can be notably different depending on the considered transmission scenario and the actual human tissue model used.

Because of the movement of the endoscope capsule, multiple on-body receiving antennas (nodes) are the generally accepted receiver structure to combat the varying channel conditions. One important design challenge at the receiver is then to exploit the available diversity. The authors in [2] studied the performance of equal gain combining (EGC) using their channel model, however only OOK is considered.

We can see from the above discussion that both bandwidth and power consumption, which also translate into fidelity at the receiver, are coupled directly with various functional blocks at the transmitter and receiver. One important task is therefore to determine the most effective combinations of the different schemes such that the best reconstruction quality can be achieved within the given budget of resources for transmission.

To the best of our knowledge, there is currently no system level assessment with respect to performance and corresponding schemes for the endoscope application. In this paper, we ensemble the entire communication chain and examine how it performs under various conditions. It is the first step in evaluating the validity of our system design, which is crucial before conducting future field tests and physical experiments. In the rest of the paper, we first describe the various blocks of the communication chain in detail in Section 2. The simulation conditions and results are presented and analyzed in Section 3. We also present a simple framework for estimating the transmit power for the capsule endoscope using UWB communication in Section 4. We then conclude the paper in Section 5 along with possible directions of future work.

\section{SYSTEM STRUCTURE}

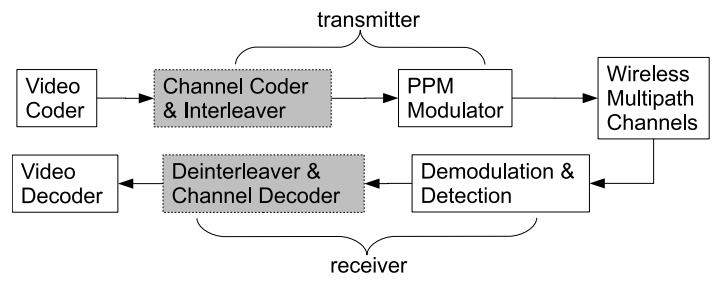

Figure 1: The Complete Communication Chain

The end-to-end communication system is depicted in Figure 1. In the following subsections we describe the components that constitute the transmitter, the channel and receiver in detail.

\subsection{Transmitter}

As mentioned in the introduction, better fidelity can be achieved by increasing the number of pulse positions if we let the bandwidth grow in accordance. However, when the symbol power is comparable to the noise level, even small deviations due to noise may lead to large decoding errors. The reason is that PPM introduces so-called threshold effects (see [1, pp. 627-631] for details) when the channel deteriorates from the optimal operation point. When threshold effects occur, on average about half of the transmitted bits will be erroneously detected. In order to make the transmission more robust and at the same time keep the transmit power efficiency provided by PPM, interleaving and channel coding can be a feasible option. By adding controlled bit level redundancy, bit errors can be detected and corrected at the receiver. Because the bit errors are bursty, an interleaver is necessary to spread the bit errors over multiple channel codewords, to improve error correction at the decoder. Note that the choice of channel codes and the size of the interleaver are both constrained by the available power and memory for processing at the transmitter. Through simulation and analysis which are presented in the Section 3, we will analyze and identify the transmission conditions when channel coding and interleaving deem necessary. We will also examine the increased fidelity provided by using a large number of pulse positions.

\subsection{Transmission Scenario and Channel Model}

Since the endoscope capsule uses the contraction of the GI tract to propel itself, its direction of movement and speed vary. Detailed and accurate modeling of the capsule movement is a rather difficult task. To gain some basic insight through simulation, we use the simplified transmission scenario illustrated in Figure 2 [5]. The patient wears a vest

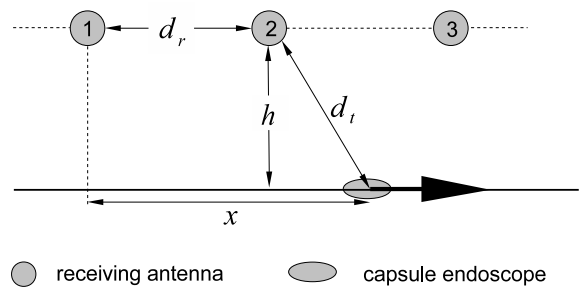

Figure 2: Simplified Transmission Scenario

that contains rows of multiple receiving nodes where each node contains a receiving antenna. The antennas on each row are placed with equal distance $d_{r}$ in between. The capsule is assumed to be transmitting on the same horizontal plane as the row of antennas is placed. The depth of the capsule is denoted by $\mathrm{h}$. The distance between the transmitter and the $i$-th receiver is then $d_{t}=\sqrt{\mathrm{h}^{2}+\left(x-(i-1) d_{r}\right)^{2}}$. where $x$ is the distance which the capsule has traveled relative to the first antenna of the row. Given the average length of the small intestine to be around $7 \mathrm{~m}$, and the transit time to be 2.5 to 3 hours [6], the average speed of the capsule is then on the order of $1 \mathrm{~mm} / \mathrm{s}$. We therefore disregard the Doppler effect, and consider the wireless channel to be slowly time varying.

Given the above described simple transmission scenario, we use the channel model that is presented in [3] with a slight modification. This model is constructed for in-body 
medical sensor placed in the abdominal region. There, the UWB channel between the transmitter and a single receiver is characterized as multipath. The channel impulse response and path loss models are presented as function of the depth of the implant relative to the body surface, where the antenna is placed. Using the illustration in Figure 2, we can extrapolate $d_{t}$ at given $d_{r}$ for each considered depth. In other words, the resulting $d_{t}$ replaces the depth parameter $\mathrm{h}$ in models from [3]. We also assume that there is always a line-of-sight (LOS) component present. The resulting channel impulse response $h(t)$ is then:

$$
\begin{aligned}
h(t) & =\delta_{0}(t)+\sum_{k=1}^{K} \beta_{k} \alpha_{k} \delta_{k}\left(t-\tau_{k}\right) \\
\alpha_{k} & = \begin{cases}1-\frac{\pi}{2}\left(\omega_{1} d_{t}^{*}+\omega_{0}\right) & k=1 \\
\left(\omega_{1} d_{r}^{*}+\omega_{0}\right) e^{(k-1)\left(\lambda_{1} d_{t}^{*}+\lambda_{0}\right)}+0.01 & k>1 .\end{cases}
\end{aligned}
$$

where $\delta_{0}(t)$ is the LOS component, $\beta_{k}$ is a binary random variable with values \pm 1 of equal probability, $d_{t}^{*}$ is $d_{t}$ normalized by $d_{0}$ and $d_{0}=10 \mathrm{~mm}$, and $\omega_{0,1}$ and $\lambda_{0,1}$ are constants used for fitting to the measurements. The average path-loss as a function of capsule's distance to antenna is:

$$
P l_{[d B]}\left(d_{t}\right)=P l_{0[d B]}+c \frac{d_{t}}{d_{0}}+\mathcal{N}\left(\mu\left(d_{t}\right), \sigma\left(d_{t}\right)\right),
$$

where $\mathcal{N}(\cdot)$ is the normal distribution, $P l_{0}, c, \mu\left(d_{t}\right), \sigma\left(d_{t}\right)$ are values given in [3]. The $\mathcal{N}(\cdot)$ component is also referred to as "shadowing".

Note that this model uses measurements averaged over a plane that covers the entire abdomen area. Therefore distinctive variations of channel characteristics in the vertical direction are not presented. Nevertheless, it is sufficient to be used as the first step in understanding and accessing the UWB transmission used by the capsule endoscope.

\subsection{Receiver}

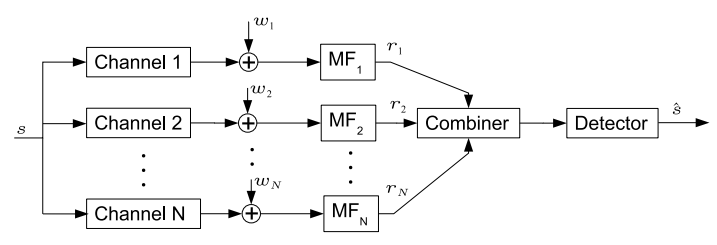

Figure 3: Receiver architecture

Figure 3 depicts the overall receiver architecture. The row of receiver nodes which are receiving from the capsule at a given time instant are shown as the $N$ receiver branches in Figure 3. Each receiver branch uses a matched filter (MF) to maximize the signal-to-noise ratio (SNR) of the received UWB signal. As we stated earlier in the introduction, because of the multiple receiver branches, diversity can be exploited to further improve the SNR. For example, the maximum ratio combiner (MRC) multiples the matched filter output by the corresponding channel gain. By doing so, the signal from each receiver branch is weighted by a factor that is proportional to its strength. Contribution from a good channel is strengthened while the poor ones are weakened. For the case of a Rayleigh flat fading channel, if the channel state information (CSI) on each receiver branch is known perfectly, the SNR per bit after combining is the sum of the

\begin{tabular}{|l|l|}
\hline \hline Sampling Frequency $F_{s}$ & $10 \mathrm{GHz}$ \\
\hline \hline Nr. of Samples per Pulse & 10 \\
\hline \hline PPM Frame Duration & $2^{M} \mathrm{~ns}$ \\
\hline \hline Maximum Channel Delay Spread & $12 \mathrm{~ns}$ \\
\hline \hline Capsule Speed & $1 \mathrm{~mm} / \mathrm{s}$ \\
\hline \hline Number of Receiving Antennas & 10 \\
\hline \hline Antenna Spacing $d_{r}$ & $10 \sim 50 \mathrm{~mm}$ \\
\hline \hline Antenna Depth $\mathrm{h}$ & $20 \sim 90 \mathrm{~mm}$ \\
\hline \hline
\end{tabular}

Table 1: Basic Parameters

instantaneous SNR of each channel [13]. The effectiveness of MRC relies on accurate estimation of CSI. A simpler alternative is the Equal Gain Combining (EGC), where signal from each branch is given equal weight and channel state estimation is therefore eliminated. We will examine the performance of different combining schemes in Section 3.

After combining, the PPM symbols are detected and converted to bit streams. They are then further processed by the de-interleaver and channel decoder for correcting bit errors, when applicable, before sent to the source decoder for reconstruction of the image frame.

\section{SIMULATIONS AND DISCUSSION}

The basic parameters we used in our simulation are presented in Table 1. Note that the maximum channel delay spread of $12 \mathrm{~ns}$ is from [3]. At the given sampling frequency of $10 \mathrm{GHz}$, using single pulse, we can safely disregard intersymbol interference when $2^{M}$ ns is the PPM frame duration and $M$ is large.

\subsection{Channel Coding and Interleaving}

We first study the impact of applying forward error correction codes. The channel code we implement at the transmitter is a simple rate $1 / 2$ convolutional code with generator polynomial $g_{0}=5, g_{1}=7$ in octal form. The encoder can be easily implemented using shift registers. We use a pseudo random interleaver of 400 bits. We compare the channel coded and interleavered PPM performance with the ones without channel coding. The combining scheme we use here is selection combining, which is explained in detail in the next section.

If we consider bandwidth (and indirectly transmit power) as the cost measure, then for the rate $1 / 2$ coded $2^{M}-\mathrm{PPM}$ it would have $2 \cdot 2^{M} / M$ number of pulse positions per source bit. In the uncoded case, if we use instead $2^{M+1}$ positions, we have $2^{M+1} /(M+1)$ positions per source bit. When $M$ is large, the bandwidth used per source bit is then roughly the same. We use this calculation of cost to compare performance from channel coded and uncoded schemes.

We first assess how the two setups compare under a not very demanding channel condition. To this end, we exclude the shadowing component of the path-loss of the multipath channel in (3). The reason for this is two fold:1) it is interesting and important to know how channel coding affects performance of PPM in other common transmission scenarios. And 2), it is easier to quantify and evaluate the result.

The simulation results are shown in Figure 4 and are presented in terms of bit error rate (BER) versus the transmit $E_{b} / N_{0}$ which is the energy per bit over the noise spectral 
density in $\mathrm{dB}$. We can see that when the $E_{b} / N_{0}$ is low, the rate $1 / 2$ channel code is not sufficient to correct the large number of bit errors. And as expected 32-PPM performs worse than 64-PPM, with shorter PPM frames. As channel condition improves, the channel code starts to correct remaining bit errors and the overall performance improves. The gain both for 128-PPM and 64-PPM is about $1.5 \mathrm{~dB}$.

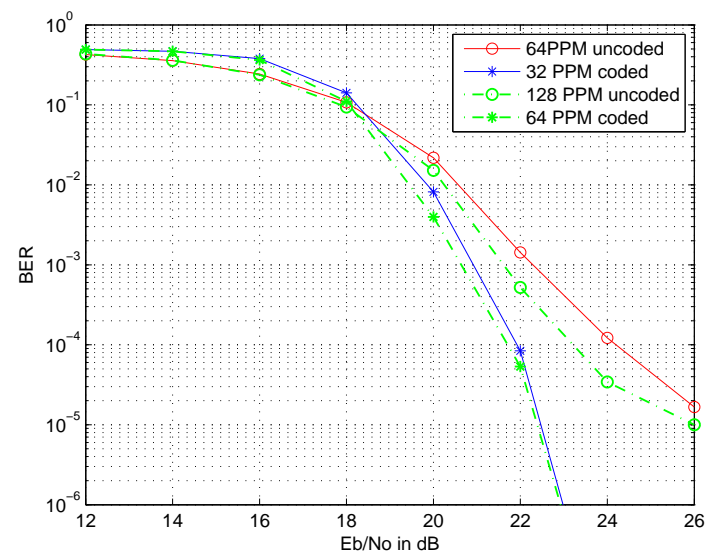

Figure 4: Performance with and without rate $1 / 2$ channel code, at $50 \mathrm{~mm}$ depth, no shadowing.

The situation becomes more complex when there is shadowing. Recall that the actual path-loss model (see (3)) of the multipath channels includes the normal distributed component $\mathcal{N}(\mu(\mathrm{h}), \sigma(\mathrm{h}))$ of certain mean and variance. This means that even when $E_{b} / N_{0}$ is considered sufficient to achieve very low BER when shadowing is not present, there is still a probability that receivers that are closer to the capsule experience heavy loss, which leads to large symbol and bit error rates. BER therefore takes on a range of values of a certain distribution. It is then insufficient to use only the average BER as the figure of merit.

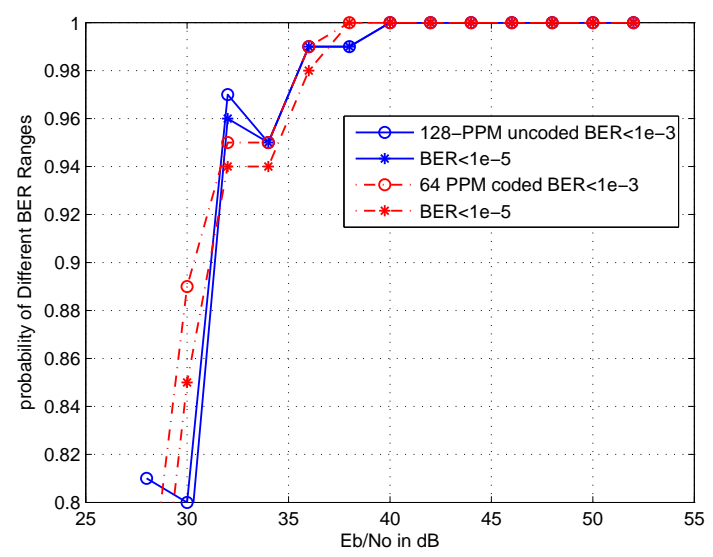

Figure 5: Performance under shadowing with and without channel coding, at $50 \mathrm{~mm}$ depth, and $50 \mathrm{~mm}$ antenna spacing.

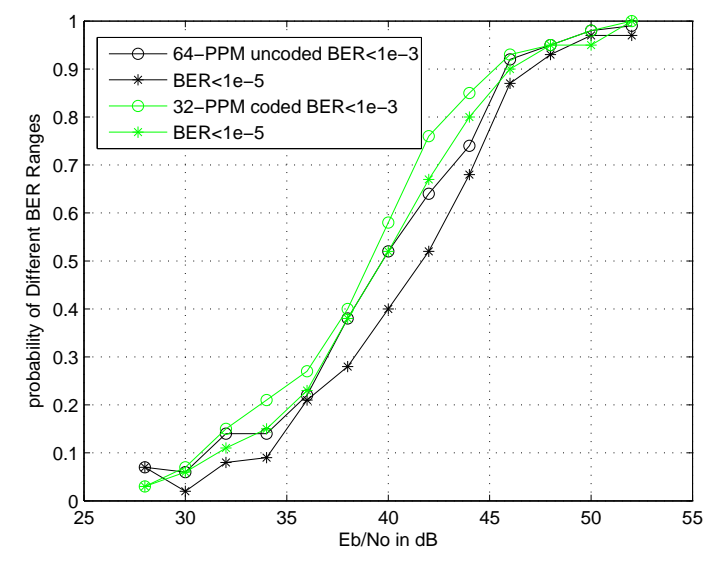

Figure 6: Performance under shadowing with and without channel coding, at $90 \mathrm{~mm}$ depth

To evaluate performance, we plot instead the probability of BER within certain range $p_{e r}$, at given $E_{b} / N_{0}$. The ranges of $\mathrm{BER}$ we consider is when $\mathrm{BER}<10^{-3}$ and $\mathrm{BER}<10^{-5}$. The motivation for the former is due to the fact that post processing (e.g., as that suggested in [14]) can be applied to the reconstructed images to tolerate bit errors up to $10^{-3}$.

Figure 5 and 6 show the performance comparison for depth $50 \mathrm{~mm}$ and $90 \mathrm{~mm}$ respectively. Note that for capsule depth of $50 \mathrm{~mm}$, the probability for the BER range is shown from above 0.8 , while the $90 \mathrm{~mm}$ drops sharply to 0 for the same $E_{b} / N_{0}$. This is expected since the greater loss in the channels is experienced with increasing depth. It is clear that with shadowing the benefit of a relatively weak channel code is no longer apparent, and the same performance can be achieved by having higher order PPM.

\subsection{Channel Estimation and Combining}

Next, we look at the effect of combining schemes. As stated in the Section 2.3, MRC requires estimation of the channel state. This can be done by transmitting pilot symbols that are known to the receiver. To estimate the channel gain, we implement the recursive least-square algorithm typically used in zero-forcing linear equalizers [13]. Instead of estimating the entire impulse response of the multi-path channel, we are only interested in the loss incurred on the LOS component which is more reliable against noise, and hence a better representative of the channel gain. In addition, we select only the three largest channel gains and use them as the weighting factor in the combining scheme, a.k.a. selection combining (SLC). This is motivated by the fact that for channels experiencing higher path-loss, estimations from pilot symbols are also less reliable because of noise. A high weighting factor (which is also incorrect) from a noisy channel will compromise the overall SNR after combining.

Figure 7 shows the performance comparison between SLC and MRC, with $50 \mathrm{~mm}$ spacing between antennas, for multipath channels without shadowing. For MRC, the corresponding channel gains are perfectly known to the receiver; whereas for SLC the gains are estimated using pilot symbols which occupy $0.5 \%$ of all transmitted symbols. In addition, we also show the results of EGC, which is the average of the 


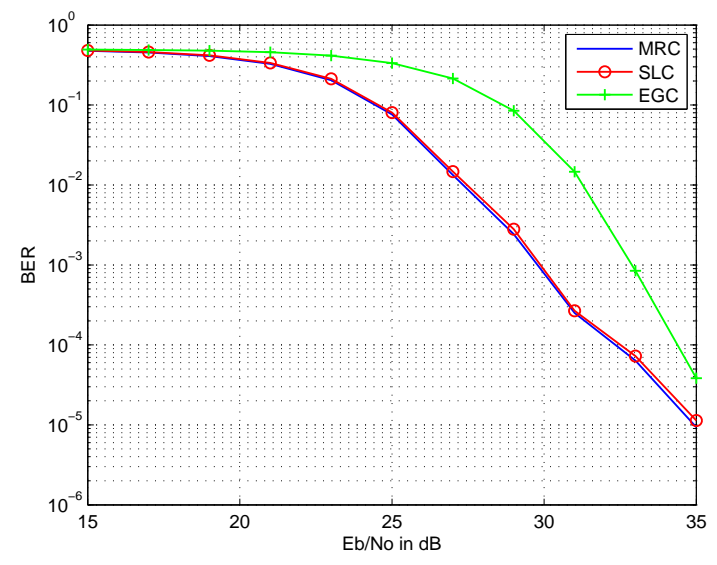

Figure 7: Performance comparisons of different combining schemes with $64-\mathrm{PPM}$, at $50 \mathrm{~mm}$ depth, $50 \mathrm{~mm}$ antenna spacing, no shadowing

estimated SLC channel gains. It is apparent from the figure that the difference between MRC and SLC is negligible while EGC suffers a significant loss.

\subsection{Overall Performance}

To summarize, when using UWB communication for capsule endoscope type application, one suitable transmission system structure that delivers the desired performance within the constraints is as follows. At the transmitter side, high order PPM modulation without channel coding can be sufficient; while for the receiver, matched filter can be applied at each receiver branch, followed by channel gain estimation and selection combining to exploit receiver diversity. The overall effect of this setup is shown in Figure 8 for $50 \mathrm{~mm}$ spacing between antennas. Here, the probability of $\mathrm{BER}<10^{-3}$ and $\mathrm{BER}<10^{-5}$ are shown over a range of $E_{b} / N_{0}$ for uncoded 128-PPM with SLC, compared to that of 16-PPM. The clear advantage of the higher order PPM is that the energy per bit required in achieving the same range of BER is considerably smaller, compared to the lower order one. As stated in the introduction, power consumption is a crucial design aspect for endoscope type in-body sensors. Using a pulse template matched to the convolution between the transmitter pulse and the channel response, as that suggested in [11], may also further improve the receiver performance. In the next section, we take a closer look at the necessary transmit power of the system, and further validate the power saving feature of high order PPM.

\section{ESTIMATION OF NECESSARY TRANS- MIT POWER}

There are two main factors that will affect the minimum required transmit power: 1) The sensitivity of the UWB receiver. 2) The received $E_{b} / N_{0}$ necessary to achieve a certain target BER for a chosen modulation scheme. In this section, we derive the transmit power $P_{T x}$ in terms of these two factors and see how the specific transmission parameters influence the power required. We emphasize that the framework we present has simplifications and details of many as-

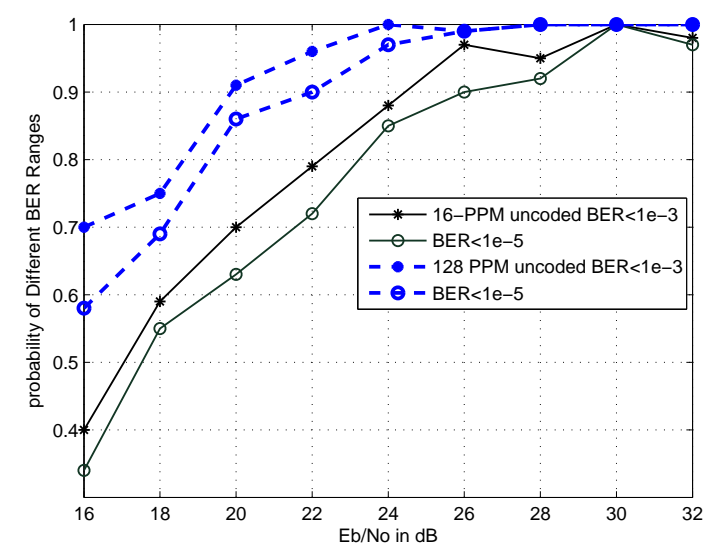

Figure 8: Performance comparisons of uncoded 128PPM and uncoded 16-PPM, with shadowing at 20mm depth.

pects that can be taken into consideration remain unknown to date. Nonetheless, we can get an idea of how transmit power may be for our application. We also state the necessary assumptions during our derivation.

We begin our analysis with the receiver sensitivity $S$ [12]. It is defined as the necessary input power the receiver needs in order to detect a signal. It can be calculated using $S=$ $k T_{e} B \mathrm{SNR}_{o}$, where $k$ is the Boltzmann's constant, $T_{e}$ is the equivalent noise temperature at the receiver, $B$ is the transmission bandwidth and $\mathrm{SNR}_{o}$ is the required $\mathrm{SNR}$ at the output of the receiver. If we consider the relation in terms of received power $P_{R x}$ (input power to the receiver) and the received noise power $P_{N}$, we can rewrite $S$ as:

$$
S=k T_{e} B \frac{P_{R x}}{F P_{N}}
$$

where $F$ is the noise figure which accounts for reduction in SNR through the receiver, and $F=P_{R x} /\left(P_{N} \mathrm{SNR}_{o}\right)$.

Because of the absorption ability of the human tissue, we assume the noise experienced in transmission is mainly due to the thermic noise coming from the antennas and receiver electronics. The received noise power $P_{N}$ can therefore be expressed as $P_{N}=k T_{e} B$, where $k T_{e}$ constitutes the thermic noise density. $S$ can then be simplified to $P_{R x} / F$. In other words, given $F$, the received power $P_{R x}$ is directly proportional to the receiver sensitivity $S$.

The next step is to determine the relationship between the transmit power $P_{T x}$ and the received power $P_{R x}$. To this end, we use the well known Friis transmission equation [7], presented here in the form which takes the large bandwidth of UWB communication into account.

$$
\frac{P_{R x}}{P_{T x}}=G_{R x} G_{T x} \frac{1}{P l\left(d_{t}\right)}\left\{\frac{\left(4 \pi d_{t}\right)^{2} \int_{f_{1}}^{f_{2}} f^{2} d f}{\overline{c_{t}}\left(f_{2}-f_{1}\right)}\right\}^{-1} .
$$

The term $\{\cdot\}$ is the so-called free space path-loss, assuming $d_{t} \gg \lambda(f)$, where $\lambda(f)$ is the wavelength of the electromagnetic (EM) wave which varies depending on the frequency. This assumption is reasonable when $d_{t}$ is above $20 \mathrm{~mm}$, since, as we shall show later, that corresponding $\lambda(f)$ is typically less than $8 \mathrm{~mm}$. The path-loss is averaged over 
the effective frequency band $\left[f_{1} \sim f_{2}\right]$, which is particularly important for UWB communication. The $d_{t}$ is again the distance between the transmit and receive antenna and $\overline{c_{t}}$ is the average speed of EM wave traversing the tissues. Because our transmission is no longer taking place in free space, we also include the path-loss term $\mathrm{Pl}\left(d_{t}\right)$, to account for the loss in power due to absorbtion of the human tissue. $G_{T x}$ and $G_{R x}$ are antenna gains at the transmitter and receiver, respectively. The antenna gain is defined as the ratio between the power needed for an ideal lossless isotropic antenna and the actual antenna applied, while achieving the same field strength. Assuming that we use an omnidirectional antenna inside the body, we can approximate the antenna gain at the transmitter to be $G_{T x} \approx 1$. Better designed receiver antennas can provide a higher gain.

By substituting (5) into (4), and solving for $P_{T x}$, we get:

$$
P_{T x}=S \cdot P l\left(d_{t}\right)\left\{\frac{\left(4 \pi d_{t}\right)^{2} \int_{f_{1}}^{f_{2}} f^{2} d f}{{\overline{c_{t}}}^{2}\left(f_{2}-f_{1}\right)}\right\} \frac{F}{G_{R x}} .
$$

This relationship describes the necessary transmit power dictated by the physics of the wireless channel (the path-loss $P l\left(d_{t}\right)$ term) ) and the physics of the transmitting and receiving antenna $\left(G_{T x}\right.$ and $\left.G_{R x}\right)$ as well as the quality of the receiver $(F)$.

To assess the required transmit power from a communication perspective, we need to link the transmit and received power with received $E_{b} / N_{0}$. Given the channel bandwidth $B$ in $\mathrm{Hz}$, the net transmission rate $R$ in bps (bit per second) and the coding gain $C$, the received noise power $P_{N}$ is the same as $N_{0} B$, since $N_{0}$ is the noisy spectral density. If we multiply $E_{b}$ in joules per bit with $R / C$ in bps, we have the received power $P_{R x}$. We arrive at the following equation:

$$
\frac{E_{b}}{N_{0}} \frac{R / C}{B}=\frac{P_{R x}}{P_{N}}
$$

where $P_{N}$ again is the received noise power. By substituting (5), and $P_{N}=k T_{e} B$ into (7), and rearranging, we get:

$$
P_{T x}=\frac{E_{b}}{N_{0}} P l\left(d_{t}\right)\left\{\frac{\left(4 \pi d_{t}\right)^{2} \int_{f_{1}}^{f_{2}} f^{2} d f}{{\overline{c_{t}}}^{2}\left(f_{2}-f_{1}\right)}\right\} \frac{k T_{e} F}{G_{R x}} \frac{R}{C} .
$$

For a desired BER, there is a corresponding $E_{b} / N_{0}$ for a given modulation and transmission scheme (see for example those described in Section 3). We can then use (8) to calculated the required transmit power. Upon close inspection of (8), we can see that the needed transmission power can be reduced by increasing the PPM alphabet (reducing $E_{b} / N_{0}$ ), increasing $G_{R x}$ and introducing coding gain $C$. Keep in mind that the processing power of coding should not override what we gain in reduced transmission power.

In the following, we present numerical examples which can help us gain further insight of the required transmit power of our system.

Example 1: Required Transmit Power based on Receiver Sensitivity. Since the optimal UWB transmitter and receiver are yet to be established for the capsule endoscope, we select parameter values from existing literature which may be suitable for our application. For example, in [16] a high data rate close range UWB receiver was implemented to have measured receiver sensitivity of $S=-80 \mathrm{dBm}$ for transmission rate of $100 \mathrm{Mbps}$ using BPSK with BER of $10^{-3}$. The reported noise figure $F$ is between 3.3 to $5 \mathrm{~dB}$. In [15], specially designed antenna for in-body communication offers antenna gain $G_{R x}$ around $6 \mathrm{~dB}$. We consider UWB transmission at $5.5 \mathrm{GHz}$ with bandwidth of $500 \mathrm{MHz}$. In order to evaluate the average free space path-loss, we refer to [8] which provides data on human tissue for electromagnetic waves in the range of 10 to $100 \mathrm{GHz}$. Consider for example the small intestine, the average speed of the $\mathrm{EM}$ wave for the $5.5 \mathrm{GHz}$ to $6 \mathrm{~Hz}$ range is $4.19 \mathrm{e} 7 \mathrm{~m} / \mathrm{s}$. We can then calculate $P_{T x}$ using (6), and plot the result as a function of depth $d_{t}$ in Figure 9. Note that the path-loss $P l\left(d_{t}\right)$ we use is the worst case value given in [3], instead of the mean.

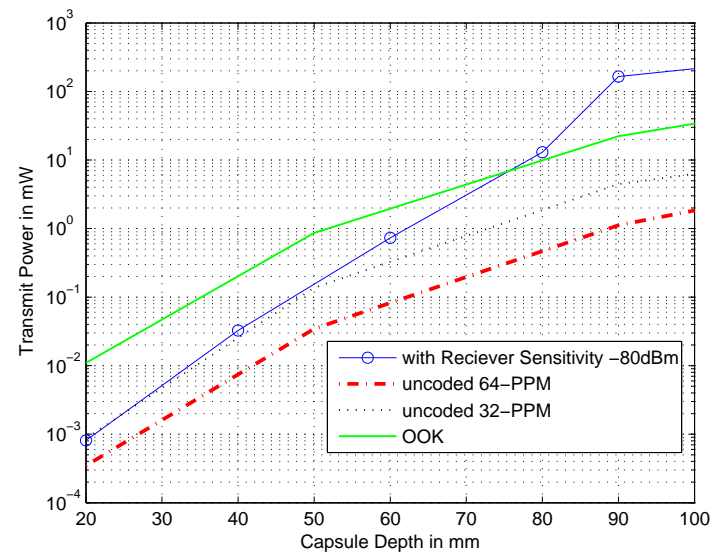

Figure 9: The estimated necessary $P_{T x}$ in terms of receiver sensitivity and modulation schemes.

Example 2: Required transmit power based on desired $B E R$. For a target BER of $10^{-3}$, we want to estimate the transmit power for $2^{M}$-PPM. We disregard the coding gain $C$ in our calculation since as we have shown earlier in Section 3.1 that there is no clear benefit in applying the rate $1 / 2$ channel code when there is shadowing. We determine the required $E_{b} / N_{0}$ for uncoded 32 and $64-\mathrm{PPM}$, by running simulations using the setup that is summarized in Section 3.3 , at different capsule depths. Note that shadowing is always considered here, since we want to estimate the transmit power as closely as possible, to that from the actual transmission scenario. Keep in mind that the $E_{b} / N_{0}$ shown in Section 3 have already taken path-loss $P l\left(d_{t}\right)$ into account.

For the purpose of benchmarking, we also include OOK. Because of the much shorter frame duration of OOK compared to the maximum channel delay spread (12ns), intersymbol interference might occur. For the sake of simplicity we assume perfect equalization at the receiver, while like 64-PPM, no channel coding is used at the transmitter. And the noise temperature $T_{e}$ is set to be $310 \mathrm{~K}$. Using (8), the resulting transmit powers are again plotted in Figure 9.

Our numerical examples show that in general, it is the receiver sensitivity that lower bounds the necessary transmit power. At shallow to medium capsule depth, higher order PPM can be a feasible modulation scheme for UWB communication. And Compared to OOK, there is a clear reduction in transmit power. The estimated power for 64-PPM is also considerably smaller than those referred in [9], [10], which assume general narrow band communication without any specific channel model. In addition, the depth of the capsule also plays an important role. Setting the transmit 
power level for the worst case scenario at all times certainly leads to poor energy efficiency. Power adaptation, on the other hand, although has clear benefits, requires additional receiver functions in the capsule which results in added processing power. A tradeoff must be carefully considered.

We also emphasize that the transmit power estimation shown here might be optimistic. There are loss factors can contribute to higher power consumption, for example, loss due to impedance mismatch between antenna and body. Another factor is the multipath components that become significant at large depth. By having an antenna with a larger gain we may compensate for some of these additional loss factors. Note also that we assume frequency flat response of the antennas. Proper modeling of antenna response in frequency and phase variations should be taken into account for better power estimation.

\section{CONCLUSIONS AND FUTURE WORK}

Because of the very low transmit power, high data rate and robustness associated with using PPM in ultra wideband communication, it is a particularly attractive communication strategy for the capsule endoscope application. In this paper, we present and analyze a complete transmission system. In particular, we study the impact of channel coding and interleaving at the transmitter, channel estimation and combining at the receiver, while incorporating a realistic transmission scenario and channel model which is established for capsule endoscope type application using UWB. We found that channel gains can be accurately estimated using a few pilot symbols. With the estimated channel gains, selection combining of the few best antennas can work as well as maximum ratio combining with perfect channel knowledge in terms of BER.

We also evaluated the impact of simple rate $1 / 2$ convolutional code with short interleaver at the transmitter. Benefit of channel coding and interleaving is clear when there is no shadowing present in the multipath channels. When there is Normal distributed shadowing, advantage of channel coding and interleaving become negligible, while the same performance can be obtained by uncoded PPM using the same amount of bandwidth. We note that a far higher $E_{b} / N_{0}$ ratio is needed to ensure that most of the BER values are below a certain threshold.

Last but not the least, we provide a simple framework for calculation of the average transmit power, both in terms of physical and hardware constraints and impact of transmission schemes. With common parameter values used in UWB communications, our estimation showed that higher order PPM is a feasible modulation scheme which consumes less power than OOK.

To further improve the robustness of the system, the source coder should be taken into account. A robust source coder can further reduce the burden on channel coding and interleaving, by coping with bit errors more effectively at the decoder. To this end, we propose error concealment and post processing schemes in [14], which are shown to be beneficial for capsule endoscope applications. Meanwhile, bandwidth efficiency of PPM can also be improved by transmitting more than a single unipolar pulse. How to combine and optimize modulation combined with error correction coding is part of our ongoing work.

\section{REFERENCES}

[1] J. M. Wozencraft and I. M. Jacobs, Principles of Communication Engineering. New York: John Wiley \& Sons, Inc, 1965.

[2] D. Anzai, S. Aoyama and J. Wang, "Performance Analysis on Equal Gain Combining Diversity Receiver for Implant Body Area Networks", International Symposium on Applied Sciences in Biomedical and Communication Technologies, ISABEL 2011.

[3] S. Støa, R.Chavez-Santiago, I. Balasingham,"An Ultra Wideband Communication Channel Moel for the Human Abdominal Region", Proc. of IEEE Globcom 2010.

[4] S. Støa, R. Chavez-Santiago, I. Balasingham, "An Ultra Wideband Communication Channel Model for Capsule Endoscopy", International Symposium on Applied Sciences in Biomedical and Communication Technologies, ISABEL 2010.

[5] G. Gjervik "Low power UWB signaling over a time varying multipath channel using multiple receiver antennas". Master thesis, Dept. of Electr. and Telecomm. NTNU, June 2011.

[6] M. Camilleri, L. J. Colemont, S. F. Phillips, M.L.Brown,G. M. Thomforde, N. Chapman, and A. R. Zinsmeister "Human gastric emptying and colonic filling of solids characterized by a new method", American J. of Physiology, vol. 257, nr. 2, p284-290, Aug. 1989.

[7] H. T. Friis, "A note on a simple transmission formula," PROC. I.R.E., vol. 34, p. 254; May, 1946

[8] C.Gabriel, "Compilation of the dielectric properties of body tissues at RF and microwave frequencies", Report N.AL/OE-TR-1996-0037, Occupational and environmental health directorate, Radiofrequency Radiation Division, Brooks Air Force Base, Texas USA, June 1996. http://niremf.ifac.cnr.it/tissprop/htmlclie/htmlclie.htm.

[9] M-C. Lin, L-R. and Dung P-K. Weng, "An ultra-low-power image compressor for capsule endoscope", BioMed. Eng. Online vol.5, nr. 14, 2006.

[10] X. Xie, G. Li, X. Chen, X. Lin and Z. Wang,"A Low-Power Digital IC Design Inside the Wireless Endoscopic Capsule", IEEE J. of Solide-state Circuits vol. 41, no. 11, pg 2390-2400, Nov. 2006.

[11] A. Khaleghi, R. Chávez-Santiago, and I. Balasingham, "Ultra-wideband pulse-based data communications for medical implants", IET Journal on Communications, vol. 4 nr.15, pg 1889-1897, 2010.

[12] FCC - National Communication Systems Technology and Standards Division, "Telecommunications, Glossary of Telecommunication Terms", General Services Administration, Information Technology Services, Aug. 1996

[13] J. Proakis, M. Salehi,"Digital Communications, 5th Edition, McGraw-Hill Science/Engineering/Math, 2007

[14] A. N. Kim, E. J. Daling, T. A. Ramstad and I. Balasingham, "Error Concealment and Post Processing for the Capsule Endoscope", Submitted to 7th International Conference on Body Area Networks (BodyNets), 2012.

[15] Q. Wang,R. Hahnel, H. Zhang and D. Plettemeier, "Wearable Vivaldi UWB Planar Antenna for In-body Communication", International Symposium on Applied Sciences in Biomedical and Communication Technologies, ISABEL 2011.

[16] F. Lee,"Energy Efficient Ultra-Wideband Radio Transceiver Architectures and Receiver Circuits" PhD Thesis, Dept. of Electrical Eng. and Comp. Sci., MIT, 2007. 